\title{
Efficient down-regulation of glia maturation factor expression in mouse brain and spinal cord
}

\author{
Smita Zaheer, Yanghong Wu, Xi Yang, Ramasamy Thangavel, Shailendra K Sahu, and \\ Asgar Zaheer \\ Department of Neurology, The University of lowa, lowa City, lowa and Veterans Affair Health \\ Care System, lowa City, lowa USA
}

\begin{abstract}
Long-lasting siRNA-based down-regulation of gene of interest can be achieved by lentiviral-based expression vectors driving the production of short hairpin RNA (shRNA). We investigated an attractive therapeutic approach to target the expression of proinflammatory GMF by using lentiviral vector encoding GMF-specific shRNA to reduce GMF levels in the spinal cord and brain of mice. To determine the effect of GMF-shRNA on GMF protein levels, we performed quantitative ELISA analysis in brain and in thoracic, cervical and lumbar regions of spinal cord from mice followed by GMF-shRNA (G-shRNA) or control shRNA (C-shRNA) treatments. Our results show a marked reduction of GMF protein levels in brain and spinal cord of mice treated with GMF-shRNA compared to control shRNA treatment. Consistent with the GMF protein analysis, the immunohistochemical examination of the spinal cord sections of EAE mice treated with GMF-shRNA showed significantly reduced GMF-immunoreactivity. Thus, the downregulation of GMF by GMF-shRNA was efficient and wide spread in CNS as evident by the significantly reduced levels of GMF protein in the brain and spinal cord of mice.
\end{abstract}

\section{Keywords}

Glia maturation factor (GMF); Short hairpin RNA (shRNA); Lentiviral vector; Gene silencing; Experimental autoimmune encephalomyelitis (EAE)

\section{INTRODUCTION}

\begin{abstract}
We have shown earlier (Zaheer et al., 2007b, Zaheer et al., 2007c, Zaheer et al., 2008) that glia maturation factor (GMF) plays a key role in regulating immune responses in the central nervous system (CNS) by way of proinflammatory cytokines/chemokines production and regulating the cytokine mediated pathways. In addition, we have also demonstrated that GMF-deficient mice failed to develop experimental autoimmune encephalomyelitis (EAE), an animal model of multiple sclerosis (MS), induced by the encephalitogenic MOG 35-55 peptide (Zaheer et al., 2010, 2011). Our earlier studies (Zaheer et al., 2007a, Zaheer et al., 2007c, Zaheer et al., 2008, Zaheer et al., 2010, 2011) clearly indicated that GMF plays a critical role in immune regulation and progression of CNS inflammation. Our long-term goal is to down-regulate the deleterious effects of GMF in the CNS thereby preventing the propagation of CNS inflammation leading to a successful GMF-inhibitor-based therapy for MS; therefore, efficient and sustained silencing of GMF expression in vivo is critical.
\end{abstract}

Address correspondence to: Asgar Zaheer, Department of Neurology, The University of Iowa, 200 Hawkins Drive, Iowa City, IA 52242, Tel. 319-353-6094; Fax 319-335-6821 asgar-zaheer@uiowa.edu. 
Sequence-specific inhibition of gene expression by double-stranded RNA is referred to RNA interference (RNAi). RNA interference can be brought about by either long double-stranded or by short interfering (si) RNAs. Recently, short interfering RNAs (siRNA) have been successfully used for sequence-specific inhibition of targeted gene expression in cultured cells and in vivo. In this study, we have examined the potential for an efficient and sustained down-regulation of GMF expression in the CNS by injecting GMF-specific siRNA and demonstrated a significant reduction of shRNA-mediated GMF expression in the spinal cord and brain of injected mice. Our overall data demonstrate that RNAi can achieve specific GMF silencing in vivo.

\section{EXPERIMENTAL PROCEDURES}

\section{Reagents}

Myelin oligodendrocyte glycoprotein peptide 35-55, complete Freund's adjuvant and pertussis toxin were purchased from Sigma-Aldrich, St. Louis, MO. Adenovirus constructs were prepared at the University of Iowa Gene Transfer Vector Core as described earlier (Davidson et al., 1993, Lim et al., 1998), using a replication-defective adenovirus (serotype 5 ) vector encoding the reporter gene for bacterial $\beta$-galactosidase.

\section{Induction of EAE}

C57BL/6 mice were purchased from Harlan Sprague Dawley, Inc., Indianapolis, IN. Mice were maintained in the animal colony at The University of Iowa and used in accordance with the guidelines approved by the IACUC and National Institutes of Health. For active induction of EAE, C57BL/6, 8-10 week-old, female mice were immunized with subcutaneous injection of $150 \mu \mathrm{g}$ myelin oligodendrocyte glycoprotein peptide 35-55 (MOG 35-55) in $100 \mu \mathrm{l}$ PBS and mixed with $100 \mu 1$ of complete Freund's adjuvant (CFA). Mice were boosted day 0 and day 2 with i.p. injection of $300 \mathrm{ng}$ pertussis toxin. Control mice received identical injections without MOG 35-55. The mice were weighed and scored daily in a double blinded fashion according to the scoring scale of 0 to 5 , grade 1 , tail paralysis; grade 2, hind limb paralysis; grade 3 , fore limb paralysis; grade 4, complete paralysis (quadriplegia); and grade 5, death.

\section{shRNA delivery in mice}

We used a lentivectors expressing GMF-specific short hairpin RNA (shRNA, Open BioSystems, Lafayette, CO, USA) that efficiently silenced expression of the GMF protein gene in primary brain cells and in mice in vivo. Hairpin mouse GIPZ lentiviral GMFshRNAmir sequence is shown:

\section{TGCTGTTGACAGTGAGCGAGGAAATCATCTTCATAGTAATTAGTGAAGCCA CAGATGTA $A T T A C T A T G A A G A T G A T T T C C C T G C C T A C T G C C T C G G A$}

Vector sequence followed by sense (GMF) sequence (underlined), loop sequence, and antisense (GMF) sequence (italic underlined). The shRNA expression lentivirus was generated as described by Xia et. al., (2004), and each mouse received i.v. injections of the lentiviral preparation $\left(1 \times 10^{11}\right.$ transduction units $)$ in $50 \mathrm{ul}$.

\section{Histological assessment}

At the peak of the disease, three mice from each experimental group were anesthetized by intraperitoneal injection of sodium pentobarbital and transcardially perfused with PBS and by $4 \%$ paraformaldehyde in phosphate buffer as described earlier (Christenson et al., 1998, Thangavel et al., 2008a, Thangavel et al., 2008b, Thangavel et al., 2009a, Thangavel et al., 2009b). The brain was removed and rinsed thoroughly with normal saline. For histochemical 
staining of $\beta$-galactosidase activity, the brains were placed in $2 \%$ paraformaldehyde and $0.2 \%$ glutaraldehyde in PBS, and then rinsed with PBS. The whole brain or coronal sections were incubated in 5-bromo-4-chloroindolyl-b-D-galactopyranoside (X-Gal) solution (1 hour at room temperature), rinsed in normal saline solution, and then post fixed with $7 \%$ buffered formalin. The fixed tissue was then processed for paraffin embedding, and sections were examined for positive staining of $\beta$-galactosidase by light microscopy.

\section{Enzyme-linked immunosorbent assay (ELISA)}

The analysis of GMF protein concentration was estimated by sandwich immuno-assay procedure as described earlier (Zaheer et al., 2007b, Zaheer et al., 2007c, Zaheer et al., 2008). Briefly, to 96-well microtiter ELISA plates pre-coated with anti-GMF capture antibodies, and samples were added and incubated overnight at $4^{\circ} \mathrm{C}$ followed by washing. Corresponding biotinylated antibodies, horseradish peroxidase-conjugated streptovidin and TMB substrate used to develop a yellow color and read by a microplate reader at $450 \mathrm{~nm}$. The concentration of GMF was estimated from a standard curve generated with each run. The lower detection limits of these ELISA are in the range of 5-10 pg/mg of protein. ELISA data are presented as mean values \pm standard deviations.

\section{Statistical analysis}

Statistical significance was assessed with one-way ANOVA followed by Tukey's procedure using SigmaStat software (SPP, Chicago, IL). A value of $p<0.05$ was considered statistically significant.

\section{RESULTS}

\section{Virus-mediated gene transfer to brain and spinal cord}

An efficient transgene expression-based strategy for treating EAE would require an efficient delivery of the therapeutic agent to CNS. Therefore, we examined transgene expression in CNS after injection of a replication-deficient recombinant adenovirus expressing $\beta$ galactosidase (AdCMV-LacZ) into the mouse. The brain and spinal cord from three mice in each group were examined 2 days after injection by histochemical staining. Histochemical analysis for $\beta$-galactosidase was carried out by incubating brain, spinal cord, or tissue sections in 5-bromo-4-chloro-indolyl- $\beta$-D-galactopyranoside (X-Gal) solution, rinsed in PBS, and fixed with formalin. Strong X-gal staining was observed on the ventral and dorsal surface of the brain along the cerebral arteries (Figure 1). Brain and spinal cord sections were examined for positive staining of X-gal (blue) by light microscopy (Figure 2 and Figure 3). These results demonstrate the widespread expression of $\beta$-galactosidase gene in brain and spinal cord of mice following i.v. administration.

\section{GMF-shRNA-mediated efficient down-regulation of GMF expression in brain and spinal cord}

Long-lasting siRNA-based down-regulation of gene of interest can be achieved by lentiviralbased expression vectors driving the production of short hairpin RNA (shRNA). We investigated an attractive therapeutic approach to target the expression of proinflammatory GMF by using lentiviral vector encoding GMF-specific shRNA to reduce GMF levels in the spinal cord and brain of mice with EAE. To determine the effect of GMF-shRNA on GMF protein levels, we performed quantitative ELISA analysis in brain and in thoracic, cervical and lumbar regions of spinal cord from mice immunized with MOG35-55 followed by GMF-shRNA (G-shRNA) or control shRNA (C-shRNA) treatments (six mice in each group). Results in Figure 4 show a marked reduction of GMF protein levels in brain and spinal cord of mice treated with GMF-shRNA compared to control shRNA treatment. The 
down-regulation of GMF by GMF-shRNA was further shown by the reduced GMFimmunoreactivity in the spinal cord sections of EAE mice treated with immunohistochemical methods (Figure 5A). The number of GMF-immunoreactive cells in MOG-immunized, and sixteen and twenty four days following GMF-shRNA treated groups are shown in Figure 5B. The GMF-shRNA treated group showed marked reduction in GMFimmunoreactive cells. We counted the GMF-positive cells on cryostat sections immunostained with GMF-antibody using $20 \times$ magnifications. The average of three separate cell counts from three mice per group was used. Thus, the down-regulation of GMF by GMF-shRNA was efficient and wide spread in CNS as evident by the significantly reduced levels of GMF protein in the brain and spinal cord of EAE mice

\section{DISCUSSION}

Adenoviral and lentiviral vectors are shown to be effective in sustained siRNA expressions in specific regions of brain (Christenson et al., 1998, Davidson et al., 2000, Davidson and Paulson, 2004, Xia et al., 2004, Zhu et al., 2004, Harper et al., 2005). Furthermore, Davidson et al., (Davidson et al., 2000) have shown sustained gene expression via adenoviral vector for 15 weeks following stereotactic intracranial delivery. Similar results were obtained using lentiviral vectors (Brooks et al., 2002). In another study, Raoul et al. (Raoul et al., 2005) demonstrated that intraspinal injection of lentiviral constructs for SOD1 siRNA in mouse led to silencing of SOD1 gene for about 120 days. Moreover, the intraspinal delivery resulted in transduction of both neurons and glia (Raoul et al., 2005). It has been shown by Dillon et al. (2005) that a sustainable silencing of gene expression could be achieved by shRNA once integrated into the genome. Lentiviral vectors have a major advantage over the other systems that they could infect post mitotic cells such as brain cells (Naldini et al., 1996a, Naldini et al., 1996b). These studies clearly demonstrate the feasibility of using viral vectors and in particular lentiviral vectors for therapy in brain.

This is the first report showing that GMF-specific shRNA is capable of efficiently inhibiting expression of GMF in vivo. In this study, we have demonstrated that GMF-shRNA mediate efficient down-regulation of GMF expression. Small interfering RNAs (siRNAs) are known to cause sequence-specific gene silencing (Mello and Conte, 2004) and therefore is ideal for silencing genes that cause various diseases of the central nervous system. Recently, gene therapy strategies were tested widely in the field of autoimmune disease (Jun et al., 2002, Bottino et al., 2003, Chernajovsky et al., 2004). In order to design an efficient strategy based on transgene expression for treating EAE/MS, it is necessary to have an efficient delivery system of the therapeutic agent to CNS. These reagents are delivered by chemically synthesized small interfering RNAs or by viral vectors carrying genes of interest. In this regard we first examined transgene expression in the CNS of the mouse after injection of a replication-deficient recombinant adenovirus expressing $\beta$-galactosidase (AdCMV-LacZ). We observed wide expression of $\beta$-galactosidase on the ventral surface of the brain of mouse following injection of AdCMV-LacZ. Histochemical analysis for $\beta$-galactosidase revealed intense staining in the major cerebral arteries and also in smaller arteries. Next, to test specific silencing of GMF in vivo, we designed shRNA construct to express a shRNA that can specifically silence GMF gene. Our data demonstrate that lentiviral-mediated delivery of RNAi can achieve specific GMF silencing in vivo. Quantitative ELISA analysis confirmed that lentiviral-mediated delivery of GMF-shRNA led to decreased levels of GMF protein in the CNS of injected mice.

In summary, we explored an attractive therapeutic approach to target the expression of proinflammatory GMF by using GMF-specific shRNA to reduce levels of GMF expression in the spinal cord and brain of mice. 


\section{Acknowledgments}

We thank Marcus Ahrens and John Newman for excellent technical help. This work was supported by the National Institute of Neurological Disorders and Stroke grants NS073670 and NS047145 (to A.Z.) and VA Merit Review award (to A.Z.).

\section{References}

Bottino R, Lemarchand P, Trucco M, Giannoukakis N. Gene- and cell-based therapeutics for type I diabetes mellitus. Gene Ther. 2003; 10:875-889. [PubMed: 12732873]

Chernajovsky Y, Gould DJ, Podhajcer OL. Gene therapy for autoimmune diseases: quo vadis? Nat Rev Immunol. 2004; 4:800-811. [PubMed: 15459671]

Christenson SD, Lake KD, Ooboshi H, Faraci FM, Davidson BL, Heistad DD. Adenovirus-mediated gene transfer in vivo to cerebral blood vessels and perivascular tissue in mice. Stroke. 1998; 29:1411-1415. discussion 1416. [PubMed: 9660397]

Davidson BL, Allen ED, Kozarsky KF, Wilson JM, Roessler BJ. A model system for in vivo gene transfer into the central nervous system using an adenoviral vector. Nature genetics. 1993; 3:219223. [PubMed: 8387378]

Davidson BL, Paulson HL. Molecular medicine for the brain: silencing of disease genes with RNA interference. Lancet Neurol. 2004; 3:145-149. [PubMed: 14980529]

Davidson BL, Stein CS, Heth JA, Martins I, Kotin RM, Derksen TA, Zabner J, Ghodsi A, Chiorini JA. Recombinant adeno-associated virus type 2, 4, and 5 vectors: transduction of variant cell types and regions in the mammalian central nervous system. Proc Natl Acad Sci U S A. 2000; 97:3428-3432. [PubMed: 10688913]

Harper SQ, Staber PD, He X, Eliason SL, Martins IH, Mao Q, Yang L, Kotin RM, Paulson HL, Davidson BL. RNA interference improves motor and neuropathological abnormalities in a Huntington's disease mouse model. Proc Natl Acad Sci U S A. 2005; 102:5820-5825. [PubMed: 15811941]

Jun HS, Chung YH, Han J, Kim A, Yoo SS, Sherwin RS, Yoon JW. Prevention of autoimmune diabetes by immunogene therapy using recombinant vaccinia virus expressing glutamic acid decarboxylase. Diabetologia. 2002; 45:668-676. [PubMed: 12107747]

Lim R, Zaheer A, Kraakevik JA, Darby CJ, Oberley LW. Overexpression of glia maturation factor in C6 cells promotes differentiation and activates superoxide dismutase. Neurochem Res. 1998; 23:1445-1451. [PubMed: 9814556]

Mello CC, Conte D Jr. Revealing the world of RNA interference. Nature. 2004; 431:338-342. [PubMed: 15372040]

Naldini L, Blomer U, Gage FH, Trono D, Verma IM. Efficient transfer, integration, and sustained long-term expression of the transgene in adult rat brains injected with a lentiviral vector. Proc Natl Acad Sci U S A. 1996a; 93:11382-11388. [PubMed: 8876144]

Naldini L, Blomer U, Gallay P, Ory D, Mulligan R, Gage FH, Verma IM, Trono D. In vivo gene delivery and stable transduction of nondividing cells by a lentiviral vector. Science. 1996b; 272:263-267. [PubMed: 8602510]

Raoul C, Abbas-Terki T, Bensadoun JC, Guillot S, Haase G, Szulc J, Henderson CE, Aebischer P. Lentiviral-mediated silencing of SOD1 through RNA interference retards disease onset and progression in a mouse model of ALS. Nat Med. 2005; 11:423-428. [PubMed: 15768028]

Thangavel R, Sahu SK, Van Hoesen GW, Zaheer A. Modular and laminar pathology of Brodmann's area 37 in Alzheimer's disease. Neuroscience. 2008a; 152:50-55. [PubMed: 18222045]

Thangavel R, Sahu SK, Van Hoesen GW, Zaheer A. Loss of nonphosphorylated neurofilament immunoreactivity in temporal cortical areas in Alzheimer's disease. Neuroscience. 2009a; 160:427-433. [PubMed: 19250962]

Thangavel R, Van Hoesen GW, Zaheer A. Posterior parahippocampal gyrus pathology in Alzheimer's disease. Neuroscience. 2008b; 154:667-676. [PubMed: 18486350]

Thangavel R, Van Hoesen GW, Zaheer A. The abnormally phosphorylated tau lesion of early Alzheimer's disease. Neurochem Res. 2009b; 34:118-123. [PubMed: 18437565] 
Xia H, Mao Q, Eliason SL, Harper SQ, Martins IH, Orr HT, Paulson HL, Yang L, Kotin RM, Davidson BL. RNAi suppresses polyglutamine-induced neurodegeneration in a model of spinocerebellar ataxia. Nat Med. 2004; 10:816-820. [PubMed: 15235598]

Zaheer A, Sahu SK, Wu Y, Haas J, Lee K, Yang B. Diminished cytokine and chemokine expression in the central nervous system of GMF-deficient mice with experimental autoimmune encephalomyelitis. Brain Res. 2007a; 1144:239-247. [PubMed: 17316572]

Zaheer A, Zaheer S, Sahu SK, Knight S, Khosravi H, Mathur SN, Lim R. A novel role of glia maturation factor: induction of granulocyte-macrophage colony-stimulating factor and proinflammatory cytokines. J Neurochem. 2007b; 101:364-376. [PubMed: 17250654]

Zaheer A, Zaheer S, Sahu SK, Yang B, Lim R. Reduced severity of experimental autoimmune encephalomyelitis in GMF-deficient mice. Neurochem Res. 2007c; 32:39-47. [PubMed: 17151915]

Zaheer A, Zaheer S, Thangavel R, Wu Y, Sahu SK, Yang B. Glia maturation factor modulates betaamyloid-induced glial activation, inflammatory cytokine/chemokine production and neuronal damage. Brain Res. 2008; 1208:192-203. [PubMed: 18395194]

Zaheer S, Wu Y, Sahu SK, Zaheer A. Overexpression of glia maturation factor reinstates susceptibility to myelin oligodendrocyte glycoprotein-induced experimental autoimmune encephalomyelitis in glia maturation factor deficient mice. Neurobiology of disease. 2010; 40:593-598. [PubMed: 20696246]

Zaheer S, Wu Y, Sahu SK, Zaheer A. Suppression of neuro inflammation in experimental autoimmune encephalomyelitis by glia maturation factor antibody. Brain Res. 2011; 1373:230-239. [PubMed: 21146509]

Zhu C, Zhang Y, Zhang YF, Yi Li J, Boado RJ, Pardridge WM. Organ-specific expression of the lacZ gene controlled by the opsin promoter after intravenous gene administration in adult mice. J Gene Med. 2004; 6:906-912. [PubMed: 15293349] 

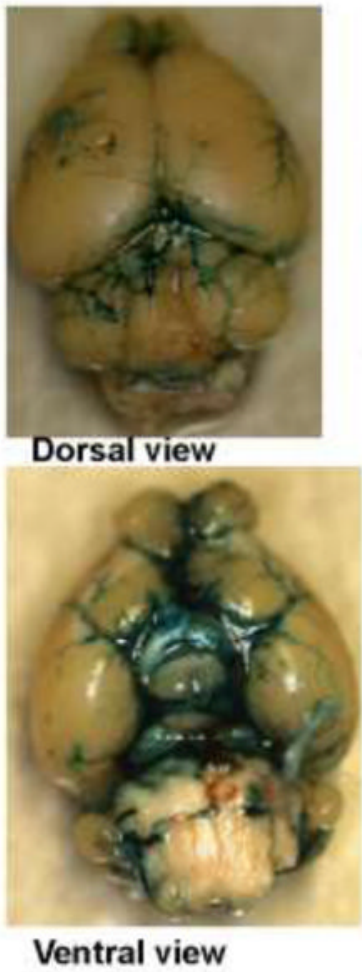

Ventral view

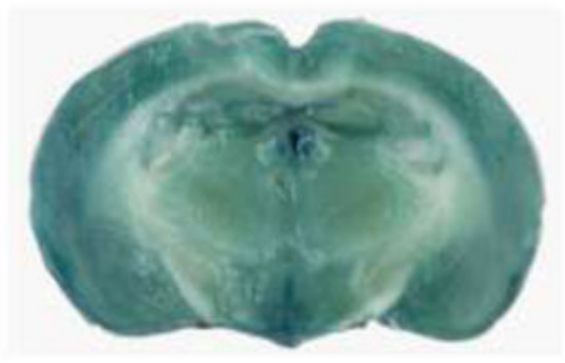

Coronal view

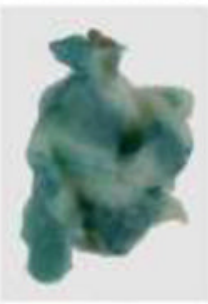

Cerebellum

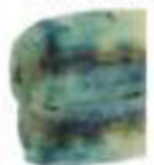

Spinal cord

Figure 1.

Expression of $\beta$-galactosidase. The ventral, dorsal and coronal views of brain of mouse $48 \mathrm{~h}$ after injection with AdCMV- $\beta$ Gal. Dark blue staining showing $\beta$-galactosidase expression on the major cerebral arteries. Transgene expression was also observed on cerebellum and spinal cord. 

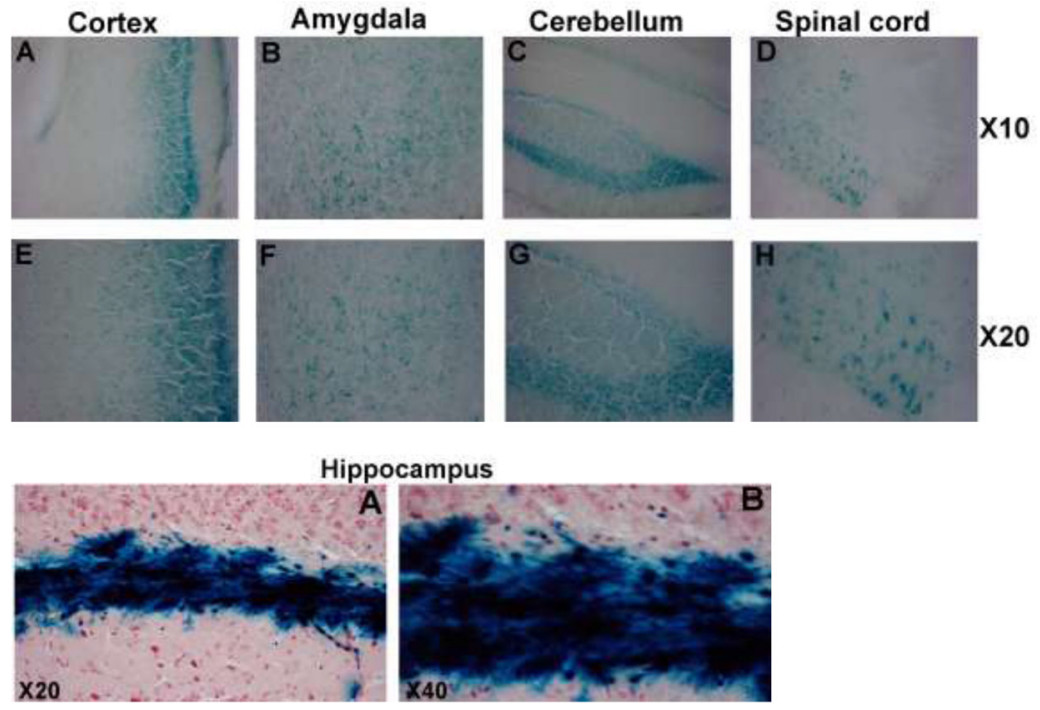

Figure 2.

Light microscopy of $\beta$-galactosidase histochemistry of mouse brain after $48 \mathrm{~h}$ after injection of the AdCMV- $\beta$ Gal shows gene expression in cortex (A, E), amygdala (B, F), cerebellum (C, G), and spinal cord (D, H). (Magnification A-D, 10×; E-H, 20x) 


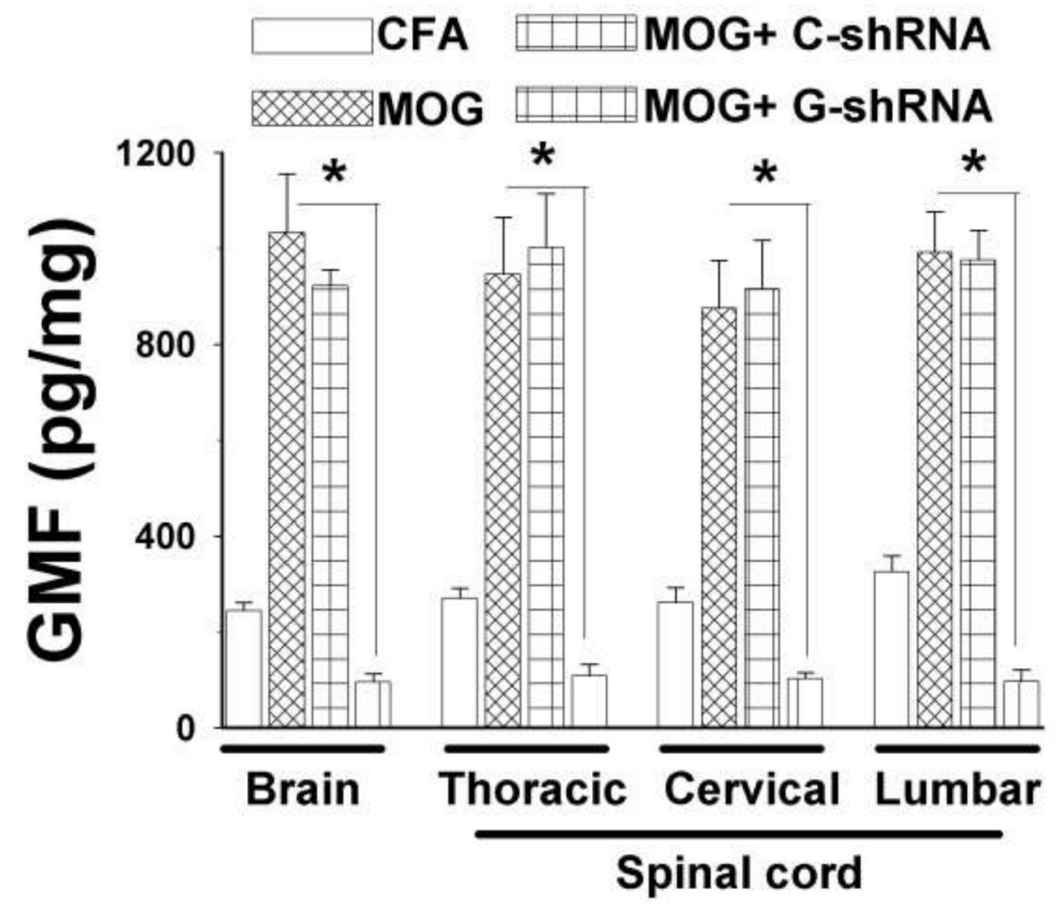

Figure 3.

Hippocampus section showing the $\beta$-gal histochemistry (counterstained with neutral red, magnification A, 20x; B, 40x). 

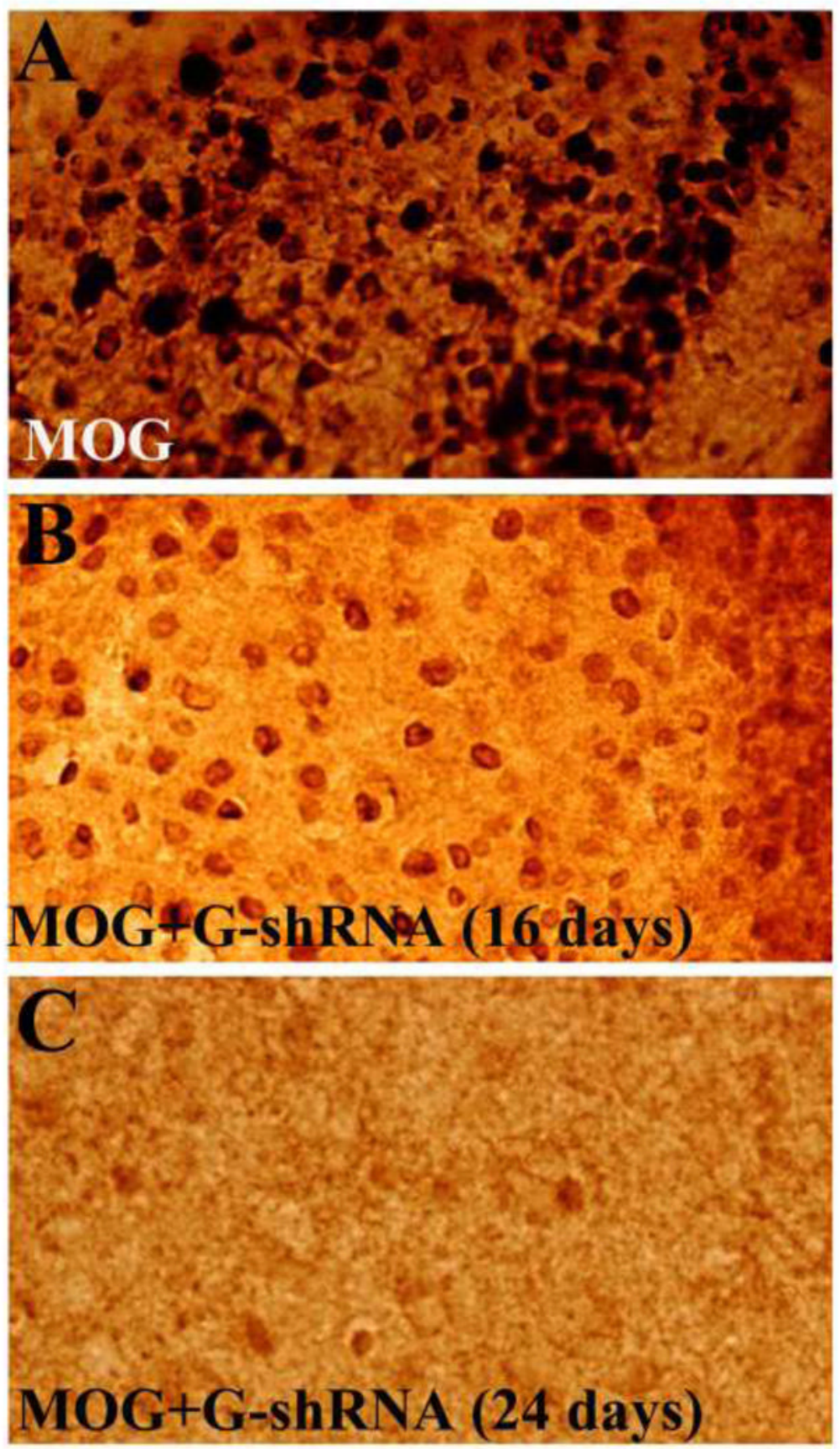

Figure 4.

LV-shRNA-mediated down-regulation of GMF in brain and spinal cord. GMF protein levels were measured by quantitative ELISA on day 24 p.i. *, $p<0.001$ for GMF-shRNA versus C-shRNA 


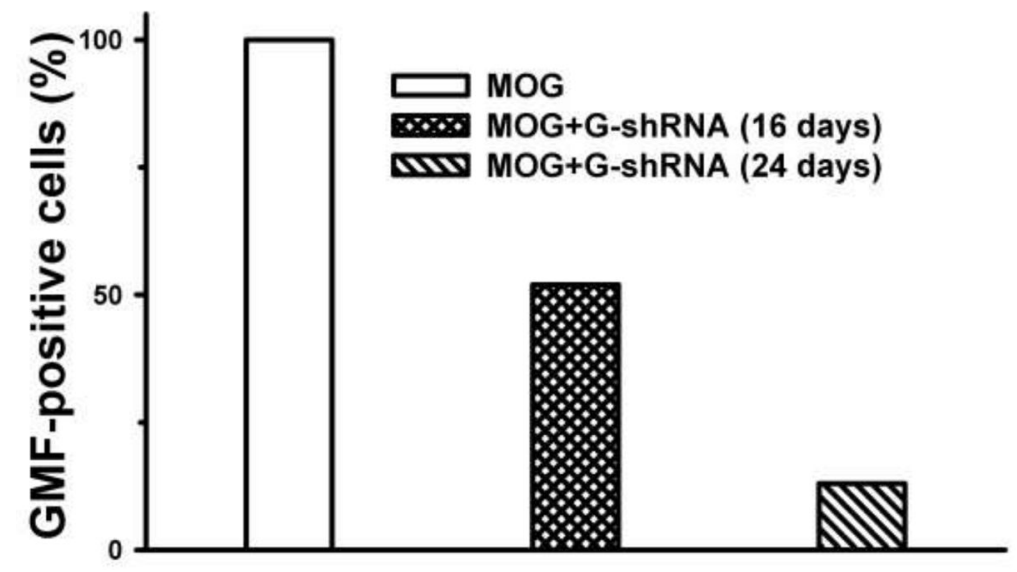

Figure 5.

(5A) Immunostaining of GMF in spinal cord sections of mice injected with GMF-shRNA. (A) MOG-immunized (day 16), (B) MOG +.G-shRNA (day 16), and (C) MOG + G-shRNA (day 24). (5B) Number of GMF-immunopositive cells in MOG-immunized, MOG + GMFshRNA (day 16), and MOG + GMFshRNA (day 24) groups. 